Aus dem physiologischen Institut in Rostock.)

\title{
Die Reaktion des Herzmuskels auf Dauerreize.
}

\author{
Von
}

\section{Laugendorff.}

\section{Die ehemische Reizung der Herzspitze.}

Die neueste Arbeit von K. K a is e r (1) über die Ursache der Rhythmicität der Herzbewegnngen nöthigt mich zu einigen Worten der Abwehr. In seiner II. Mittheilung hatte $\mathrm{Ka}$ is er allgemein zu beweisen gesucht, dass dem Herzmuskel nicht, wie vielfach behauptet wird, rhythmisehe Eigenschaften zukommen, sondern dass die unter gewissen Bedingungen zu beobachtenden Pulsationen desselben durch äussere, in jenen Bedingungen gegebene Umstände veranlasst" werden. So hatte er auch die in Folge e he mischer Reizung der abgeklemmten Herzspitze von mir beobachteten rhythmischen Pulsationen nicht als beweiskräftig anerkannt und eine von der meinen durchaus abweichende Deutung derselben gegeben. Gegen diesen Deutungsversuch hatte ich mich in einer kleinen Abhandlung: "Zur Lebre von der Rbythmicität des Herzmuskels" (2) gewendet, indem ich ihre Unhaltbarkeit durch eine Reihe neuer Versuche nachwies. Nun kommt $\mathrm{K}$ aise $\mathrm{r}$ in seiner III. Mittheilung (3) auf die chemische Reizung der Herzspitze zurück und erklärt die von mir erhobenen Einwände gegen seine Lehre für "nicht relevant". Dieses Urtheil sucht er für jeden einzelnen der von mir angegebenen Versuche zu begriuden, legt aber seiner Kritik e in e ga nz n e u e, von se in er früheren grundverschiedene Erklärung der in Rede stehenden Erscheinung zu Grunde, eine Erklärung, deren Möglichkeit in der ersten Abhandlung nicht einmal angedentet ist, gegen die sich also meine Experimente nicht hatten richten können. Um jedes Missverständniss zu vermeiden, stelle ich $\mathrm{K}$ a is e r's frübere und spätere Interpretation mit seinen eigenen Worten bier- 
her. Er sagt (II. Abldlg. S. 292) 1) : „Bringt man auf die nach Bernstein abgeklemmte, in Ruhe befindliche Herzspitze ein 4 bis 9 qmm grosses Fliesspapierstiuckchen, welches zuvor in eine concentrirte Kochsalzlösung getaucht worden war, so seben wir fast unmittelbar darauf die Herzspitze eine längere Reihe von Pulsen ausführen. Der Rhythmus dieser Pulse ist direkt abhängig von dem Rhythmus des Ventrikelrestes. Diedurch die concentrirte Kochsalzlösung in Contraction versetzte Spitze wird durch jede zweite oder dritte Contraction des Ventrikelrestes pas s iv gedehnt; bei-der Erschlaffung des Ventrikelrestes zieht sich die immer in Contraction befindliche, durch die unter bestimmtem Druck eingetriebene Blutmenge aber gedehnte Spitze wieder zusammen." Ich denke, das kann doch nur so verstanden werden, dass die Spitze in Folge der chemisehen Reizung in den Zustand der Da uercontraction geräth und nur durch die rhythmische Füllung vom übrigen Herzen her $\mathrm{ab}$ und $\mathrm{zu}$ in $\mathrm{s} \mathrm{ch}$ e in b a re Diastole versetzt wird.

Was gegen diese Deutungsweise einzuwenden sei, habe ich in meiner angefiihrten Arbeit gezeigt; es hiesse, deren Inhalt wiederholen, wollte ich meine Gegenversuche noch einmal hierhersetzen. Jetzt lässt aber $\mathrm{K}$ a i s er diese seine Erklärung stillschweigend fallen und giebt, ohne i m Gering sten dara f a u f merksam zu machen, eine durchans neue, der gegenüber meine Kritik ganz sinnlos erscheiren muss. Auch hier gebe ich seine eigenen Worte wieder. Er sagt (III. Abhandlung S. 8): „Zunächst stellte ich fest, dass, der bisherigen Annahme entgegen (! Ref.), die gesättigte Koehsalzlösung für den nervenfreien Muskel $\mathfrak{u}$ berh a ut keinen Reizbildet!" Man beachte: früher sollte die Spitze dureh Kochsalzlösung in dauerde Contraction versetzt werden! Jetzt soll das Kochsalz nur (S. 8) „die Erregbarkeit der Herzspitze erhöhen". Und weiter: "Der unter normalen Bedingnngen in der abgeklemmten, blutgefüllten Spitze vorhandene Druck genügt zur Auslösung von Contractionen nicht. Wird die Erregbarkeit der Spitze durch Auflegen eines Kochsalzkrystalles gesteigert, so wird d e r vorher ungen ügende Druck zum wirksamen Reiz und die Spitze be-

1) Die Hervorhebung der gesperrt gedruckten Sätze rührt grössten. theils von mir her. 
ginnt zu pulsiren" (S. 10 und 11). Das ist freilich etwas ganz Anderes! Hiernach handelt es sich doch um wirkliche Pulsationen!

Es wäre ja nichts dagegen zu sagen, wenn $\mathrm{K}$ a i s er erklärte: Meine späteren Ueberlegungen oder auch die Einwände von Langendorff haben mich davon uberzeugt, dass meine frühere Interpretation unrichtig ist; ich gebe jetzt eine neue und will nachweisen, dass sie durch jene Einwände nicht widerlegt wird. Aber kein Woìt davon! Der früheien Deutung wird gar nicht mehr gedacht, und meine Experimente werden so beurtheilt, als ob sie sich nicht gegen die erste, sondern gegen die neu mitgetheilte Auffassung gerichtet hätten!

"Dass die Spitzencontractionen von Aktionsströmen begleitet sind, habe ich nie bezweifelt", sagt jetzt $\mathrm{K}$ aiser. Das ist ja möglich; wenn er aber früher diese Contractionen als echte Pulse gar nicht anerkannte (s. 'o.), so hätte er doch alles Recht zu solchem Zweifel gehabt, und jedenfalls war ich berechtigt, aus der von mir bewiesenen Existenz der Aktionsströme zu schliessen, dass die erwähnten Kochsalzpulse aktiver Natur sein muissen. Wenn $\mathrm{Ka}$ is e $\mathrm{r}^{\mathrm{r}}$ dies jetzt selber voraussetzt, so hat allerdings meine Beweisführung keinen Sinn mehr. Ebenso verhält es sich mit meinen anderen Versuchen. Noch ein Beispiel! Kaiser gesteht neuerdings zu, dass zwischen der Frequenz der Zusammenziehungen der Herzspitze und denen des Ventrikelrestes ein harmon is ches Verbä lt n iss nicht besteht, ja unmöglich bestehen kann. Damit lässt er seine fruhere Behauptung fallen, die, wie schon angefuihrt, dahin ging: „Der Rh yth m us dieser (scil. scheinbaren) Palse ist direkt abhängig vondem Rhythmus des Ventrikelrestes. Die Spitze wird u r ch jede oder jede $\mathrm{z}$ weite oder d ritte Contraction des Ventrikelrestes p a s siv gedehnt u. s. w." War es unbegründet, wenn ich, was er jetzt rügt, aus diesen Sätzen das Postulat eines har m o$\mathrm{n}$ is che $\mathrm{n}$ Verhältnisses ableitete und mich damit abgab, ein solches als nicht bestehend nachzuweisen?

Jetzt möchte ich allerdings fast bedauern, Zeit und Mähe auf die Widerlegung von Behauptungen verwendet zu haben, die ihr eigener Urheber in aller Stille begräbt.

Aus diesem Grunde verzichte ich anch vorläufig daranf, näher auf die neue Meinung Kaiser's einzugehen, dass die Wirkung 
des Kochsalzkrystalls nicht auf einer Reizung sondern nur einer Erregbarkeitssteigerung des Herzmuskels beruhen soll. Doch darauf möchte ich hinweisen, dass gewiss die meisten Physiologen mit mir die Pflüger'sche Ansicht (4) theilen, dass Umstände, welche die Erregbarkeit schnell steigern, auch erregen könnẹ. Und ferner: zugegeben, das $\mathrm{NaCl}$ sei wirklich, wie es $\mathrm{K}$ aiser aus seinem Versuch am nervenlosen Sartoriusende erschliesst, kein Reiz für den Muskel; gilt dies zugleich anch für alle die übrigen Stoffe, die meinen Beobachtungen nach die abgeklemmte Spitze zum Pulsiren bringen, auch ohne, wie das $\mathrm{NaOH}$, schnell zerstörend zu wirken? Dann gäbe es ja iuberhaupt kaum noch chemische Muskelreize! Ich muss verlangen, dass Kaiser für jeden der von mir verwendeten Körper (Salzsäure, Ammoniakdämpfe, Kalkwasser, Silbernitrat, Froschgalle, gallensaure Salze, Milchsäure u. a. m.) den entsprechenden Nachweis fuilht. Bis dahin kann ich die Ueberzengung nicht fallen lassen, dass das von mir benutzte Verfaliren die Rhythmicität des Herzmuskels beweist.

\section{I1. Die Reizung des Herzmuskels mit Kettenströmen.}

Die Einwürfe Kaiser's richten sich, wie man weiss, nicht allein gegen die aus der chemischen Reizung des Herzmuskels gezogenen Schliisse, sondern auch gegen die Verwerthung der galvanischen und mechanischen Reizung der Herzspitze zu Gunsten des rhythmischen Vermögens des Herzmuskels.

Ich habe Herrn Dr. Fon robert veranlasst, die zuerst von Eckhard (5), später von Foster und Dew-Smith (6), Scherhey (7) u. A. erforschte Reaktion des Herzmuskels auf galvanische Durchströmung noch einmal und zwar mit Rücksicht auf die Bedenken Kaiser's zu untersuchen. Herr Fonrobert hat, wie er in seiner Dissertation (8) mitgetheilt, bezüglich der Wirkung von Stromesstärke und Stromesrichtung die Ergebnisse früherer Beobachter bestätigen können; insbesondere hat er auch die zuerst von Bi edermann (9) nachgewiesenen polaren Wirkungen des Stromes studirt und die von diesem Forscher gemachten Angaben dahin erweitert, dass an der durch Abquetschung isolirten Herzspitze sich nicht allein die erregende Wirkung der Kathode, sondern auch, wenn man die Spitze zum Pulsiren bringt, die hemmende Wirkung der Anode nachweisen lässt. 
Besondere Sorgfalt hat er aber der Frage zugewendet, ob aus dem rhythmischen Pulsiren der galvanisch durchströmten Spitze auf ein rbythmisches Vermögen des Herzmuskels geschlossen werden darf, oder ob die gegen diese Schlussfolgerung von $\mathrm{K}$ a i s e $\mathbf{r}$ gemachten Einwände berechtigt sind. $\mathrm{K}$ a i s er meint (a. a. 0 . S. 284), "dass auch di e s e rhythmischen Bewegungen der Herzs pitze dadurch zu Stande kommen, dass der kontinuirliche Reiz durch die Veränderungen, welche er am Herzen hervorruft, unterbrochen wird“. Die Unterbrechungen sollen „auf rein äusserlichem, künstlichem Wege" geschehen. Ich kann dies nicht anders verstehen, als dahin, dass entweder an der sich kontrahirenden Spitze Elektrodenverschiebungen stattfinden oder dass die Formveränderung des Herzens Dichtigkeitssehwankungen des Stromes hervorrufen soll.

Schon Eckhard ist dieser Auffassung durch einen, wie ich glaube, entscheidenden Versuch (Einschaltung eines Nervmuskelpräparates in den Stromkreis) entgegen getreten. Kaiser hält das Inruhebleiben des Froschschenkels nicht für beweisend, weil es sich um Stromesschwankungen von geringer Steilbeit handle, für die wohl der Herzmuskel, nicht aber der Nerv empfindlich sei.

Es sind im Wesentlichen drei Versucbsreihen, die Fonrobert zur Widerlegung der $\mathrm{K}$ ais er'schén Auffassung ausgefithrt hat; sie alle beweisen, dass von Widerstandssehwankungen in seinem Sinne nicht die Rede sein kann.

1. Die abgeklemmte Herzspitze eines mit Kurare vergifteten Frosches wird in eine viereckige, mit 0,6 procentiger Kochsalzlösung gefüllte Glaswanne versenkt, deren Schmalwände mit Elektrodenblechen bekleidet sind. Wird der Herzmuskel auf diese Weise von parallelen Fäden eines Kettenstromes durchflossen, so pulsirt er rhythmisch, wie wenn die Elektroden ihm unmittelbar angelegt worden wären.

Nimmt man an, dass der Leitungswiderstand des Herzens und der Kochsalzlösung annähernd gleich gross sei, so können die Formveränderungen des Herzens keinen Einfluss auf die Stromesdichte ausiiben, und eine Verschiebung der Elektroden ist durch die Versuchsanordnung ausgesehlossen.

2. Sind nach $\mathrm{K}$ a is er die angeblichen Stromesschwankungen auch zu langsam, um das Nervmuskelpräparat zu erregen, so 
sollen sie doch genügend steil sein, um den Herzmuskel zu reizen. Wenn das letztere der Fall ist, so müssten sie auch im Stande sein, Induktionswirkungen hervorzurufen. Das ist indess keineswegs der Fall. Schaltet man in einen starken Kettenstrom die Herzspitze und ausser ihr die primäre Rolle eines Induktionsapparates ein, so gibt ein im sekundären Kreis befindliches Nervmuskkelpräparat nur bei Sehliessung und Oeffnung des primären Stromes Zeichen von Erregung, bleibt aber während der Schliessungsdauer auch bei ïbereinander geschobenen Rollen völlig in Ruhe; der Herzmuskel pulsirt natïrlich während dieser Zeit. Die angeblichen langsamen Schwankungen müssten durch die Einschaltung des Induktionsapparates gewissermassen in steile Schwankungen transformirt sein. Dennoch bleibt die Wirkung auf den Nerv aus. Wie dieser, so lässt auch ein in den sekundären Kreis aufgenommenes empfindliches Telephon nicht die mindeste Schwankung erkennen.

3. Wenn, wie Kaiser zu glauben scheint, bei der konstanten Durchströmung des Herzens Widerstandsschwankungen im Spiele sind, so miissten diese unmerklich klein und deshalb unwirksam werden, wenn man neben dem Herzen einen Leitungswiderstand von sehr bedeutender Grösse einschaltete. Es zeigt sich aber, dass man 45000 bis $50000 \mathrm{Ohm}$ in den Stromkreis aufnehmen kann (Widerstand einer starken Zinksulfatlösung in einer Röhre von etwa $20 \mathrm{~cm}$ Länge und $1 \mathrm{~mm}$ Durchmesser), ohne die rbythmischen Pulsationen der Herzspitze aufzuheben.

Ich möchte glauḅen, dass diesen Erfahrungen gegeniiber die Ansicht $\mathrm{K}$ a i s e r' 's sich nicht halten lässt, und dass man ohne Bedenken auch fürderhin den Satz gelten lassen darf, dass der konstante Strom den Herzmusel zur rhythmischen Thätigkeit bringt.

\section{Literatur.}

1. K. $\mathrm{K}$ a is er, Untersuchungen über die Ursache der Rhythmicität der Herzbewegungen. II. Zeitschr. f. Biologie. Bd. XXX. (N. F. Bd. XII.) S. 279 .

2. O. L a g end or ff, Zur Lehre von der Rhytbmicität des Herzmuskels. Dieses Archiv Bd. 57. S. 409.

3. K. $\mathrm{K}$ a is e $\mathbf{r}$, Untersuchungen etc. III. Zeitschr. f. Biologie. Bd. XXXII. (N. F. Bd. XIV.) S. 1. 
4. E. Ṕ f 1 ü $\mathrm{g}$ e r, Die teleologische Mechanik der lebendigen Natur. Dieses Archiv Bd. 15. S. 89.

5. C. E ck ha rd, Ein Beitrag zur Theorie der Ursachen der Herzbewegung. Beitr. z. Anat. u. Physiologie. Bd. I. S. 145.

6. M. Foster und Dew-Smith, The effects of the constant current on the heart. Journal of Anatomy and Physiology. Vol. X. 1876. p. 735.

7. J. A. Seherhey, Zur Lehre von der Herzinnervation. Du Bois Rey mond's Archiv f. Physiol. 1880. S. 258.

8. A. F o n r ober t, Ueber die elektrische Reizung des Herzens. Dissertat. Rostock 1895 .

9. W. Biedermann, Beiträge zur allgemeinen Nerven- und Muskelphysiologie. XIV. Mittheilung. Ueber das Herz von Helix pomatia. Sitz.-Ber. d. k. Akad. d. Wiss. in Wien. III. Abth. Jänner 1884. S. 47 und: Elektrophysiologie. Jena 1895. I. Abtheilung. S. 219.

\section{Zur Frage der Hörfähigkeit labyrinthloser Tauben. \\ Von \\ W. Wundt.}

In einem in Band 61 dieses Archivs (Heft 1-3, S. $113 \mathrm{ff}$.) enthaltenen Aufsatze bemerkt J. B e r n s te in, nach der Exstirpation des Labyrinths bei Tauben trete erstens die Degeneration der Hörnerven unter allen Umständen ein, und sie ergreife zweitens „den grössten Theil des Oetavusstammes und zwar den $\mathrm{N}$. cochlearis vollständig". Speciell an dem von mir beobachteten Thier, an dem die in meinen Philos. Studien (Bd. IX, S. $496 \mathrm{ff}$.) mitgetheilten Hörprüfungen vorgenommen wurden, und das auf der einen Seite vor 12, auf der andern vor 6 Wochen von $E$ wald operirt worden war, "musste“, wie Bernste i n meint, „die Dege-

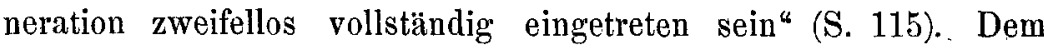
gegenüber erlaube ich mir mitzutheilen, dass nach der von Dr. H e l d längere Zeit nach der Section des Thieres ausgeführten mikroskopischen Untersuchung an den frei liegenden Enden des durchschnittenen Hörnerven nur Anfänge einer Degeneration zu finden waren, die sich noch nirgends über die primären Centren fortsetzte. Was in dem Sectionsprotocoll (a. a. 0. S. 504) über die Atrophie einzelner Hirntheile bemerkt ist, kann daher nach dem mikroskopischen Befund nur auf zufällige Abweichungen und 